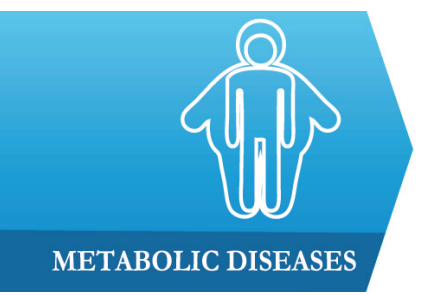

1) Graduate Program in Endocrinology, Universidade Federal do Rio Grande do Sul (UFRGS), Porto Alegre, Brazil

2) Laboratory Diagnosis Department, Hospital de Clínicas de Porto Alegre (HCPA), Porto Alegre, Brazil

3) Experimental Research Center and Endocrinology Department, Hospital de Clínicas de Porto Alegre (HCPA), Porto Alegre, Brazil

4) Diabetes and Metabolism Group, Centro de Pesquisa Clínica, Hospital de Clínicas de Porto Alegre (HCPA), Porto Alegre, Brazil
DOI: $10.15386 / \mathrm{mpr}-1743$

Manuscript received: 29.05 .2020

Received in revised form: 14.08.2020

Accepted: 30.08 .2020

Address for correspondence:

jcamargo@hcpa.edu.br

This work is licensed under a Creative Commons Attribution-NonCommercialNoDerivatives 4.0 International License

\title{
Factors associated with glycated albumin in adults without diabetes
}

Priscila Aparecida Corrêa Freitas ${ }^{1}$, Mayana Kieling Hernandez ${ }^{2}$, Joíza Lins Camargo $0^{3,4}$

\begin{abstract}
Background and aims. Glycated albumin is a glycemic marker useful in shortterm monitoring and in situations when a glycated hemoglobin test is not reliable. This study aims to evaluate glycated albumin levels and its associated factors in normoglycemic adults from Southern Brazil.

Method. 136 individuals, without diabetes or pre-diabetes, were included in this cross-sectional study. Levels of glycated albumin, glycated hemoglobin, and other biochemical markers were measured.

Results. Glycated albumin levels ranged from $11.1 \%$ to $17.5 \%\left(2.5^{\text {th }}\right.$ and $97.5^{\text {th }}$ percentiles). Glycated albumin/glycated hemoglobin ratio was $2.8 \pm 0.2$. Glycated albumin did not differ according to gender and age groups. However, in overweight individuals, levels of glycated albumin and glycated albumin/glycated hemoglobin ratio were lower and weakly and negatively correlated with body mass index.

Conclusions. Glycated albumin levels in Brazilians were similar to those previously described in other populations. Glycated albumin seems to be irrespective of gender or age, but weakly correlated with weight. These aspects should be taken into account in the interpretation of glycated albumin results.
\end{abstract}

Keywords: glycated albumin, glycated hemoglobin, diabetes

\section{Background and aims}

Currently, diabetes mellitus is one of the chronic diseases with the highest prevalence. Approximately 425 million people in the world are said to have diabetes and the trend is for increasingly larger rates [1,2]. It is estimated that almost $8.7 \%$ of the Brazilians, aged from 20 to 79 years have diabetes [1]. Hence, improving diagnosis and glucose monitoring methods are on high demand and expected from the scientific community.

Traditionally, glycated hemoglobin (A1c) is recommended for the assessment of glycemic control in all patients with diabetes. More recently, it is also a test of choice to diagnose diabetes [2]. A1c is a long-term glycemic marker strongly related to diabetic chronic complications [3]. However, its use is not recommended when interfering situations of hemoglobin metabolism are present [4]. Thereby, in cases such as anemia, iron deficiency, hemoglobinopathies, chronic renal disease and pregnancy, this marker may provide falsely increased or decreased results and should be avoided [4-6].

Glycated albumin (GA) is another potential marker in the screening/ diagnosis and monitoring of diabetes [7]. It is a glycated serum protein that has been widely studied in the last decade, since the development of a new enzymatic methodology for its analysis [7-9]. GA reflects short-term average blood glucose, it is not influenced by the clinical conditions that alter A1c results and may be more sensitive to capture variations in blood glucose levels when compared to A1c $[7,10]$. In clinical practice, GA/A1c ratio has been described as a good marker of postprandial glycemia and blood glucose variability [11]. However, GA is 
still not commonly used in clinical laboratories and there is no international consensus about its reference interval.

In the literature, several studies have determined the GA levels in populations with and without diabetes. However, most of these studies were carried out in Japan or in other Asian countries [5,12-14]. Some authors have suggested that there is an ethnic influence on glycated proteins levels [15], including the GA levels [6,16], but this issue warrants additional studies. Data providing GA normal ranges in people from other regions such as Europe [17] and Northern America [6] are already available, but no study was carried out in Southern America. Moreover, the influence of gender, weight, and age on GA values is not entirely elucidated $[5,13,18,19]$. Therefore, we aimed to evaluate the levels of GA and their associated factors in normoglycemic individuals from southern Brazil.

\section{Methods}

\section{Subjects}

Adult and apparently healthy volunteers from Porto Alegre, Southern Brazil, without a known history of diabetes or prediabetes (fasting glucose $<100 \mathrm{mg} / \mathrm{dL}$, and $\mathrm{A} 1 \mathrm{c}<5.7 \%$ ) [2], and without using oral antidiabetic agents were enrolled in this study. We excluded participants who, by self-report, indicated any comorbidity or condition that could affect A1c or GA levels, such as hypothyroidism, hyperthyroidism, cirrhosis, pregnancy, erythropoietin treatment or any other chronic condition. We also excluded those individuals with increased risk for kidney disease by glomerular filtration rate (if $<60$ $\mathrm{mL} / \mathrm{min} / 1.73 \mathrm{~m}^{2}$ ), anemia (if hemoglobin $<13.0 \mathrm{~g} / \mathrm{dL}$ for male, or $<12.0 \mathrm{~g} / \mathrm{dL}$ for female), or with abnormal serum albumin concentration $(<3.0 \mathrm{~g} / \mathrm{dL}$ and $>5.0 \mathrm{~g} / \mathrm{dL})$. This cross-sectional study was disclosed by social media for recruitment of volunteers and was approved by the local Ethics Committee, under number GPPG 13-0440. All participants signed the informed consent beforehand.

\section{Blood sampling}

The volunteers underwent clinical evaluation and had peripheral blood samples collected in the morning after an overnight $8 \mathrm{~h}$-fasting. Two specimens of blood were obtained: one preserved in K2EDTA (BD Vacutainer ${ }^{\circledR}$ EDTA K2 $4 \mathrm{~mL}$, Becton Dickinson, Franklin Lakes, NJ) and one without anticoagulant (BD SST $^{\circledR}$ II Advance ${ }^{\circledR} 5 \mathrm{~mL}$, Becton Dickinson, Franklin Lakes, NJ). Serum was obtained from the second sample by centrifugation $(3000 \times \mathrm{g}, 10 \mathrm{~min})$ no longer than 30 minutes after venipuncture. All samples were frozen at $-80^{\circ} \mathrm{C}$ and thawed only once at $4^{\circ} \mathrm{C}$ temperature on the day of the analyses.

\section{Laboratory analysis}

GA was determined in serum samples using an
California, USA). GlycoGap ${ }^{\circledR}$ GA assay quantifies the total of glycated serum proteins (GSP $\mu \mathrm{mol} / \mathrm{L}$ ) and the following math equation converts them into GA $\%=(\mathrm{GSP}$ $\mathrm{x} 0.182+1.97 /$ total albumin) $\mathrm{x} 100$ [20]. The intraassay repeatability of GlycoGap ${ }^{\circledR}$ kit in our laboratory was $3.5 \%$ [21]. We calculated the GA/A1c ratio, using levels of A1c converted to "\%", as recommended by National Glycohemoglobin Standardization Program (NGSP). Serum samples were also used to measure total albumin by bromocresol green colorimetric method; creatinine by Jaffe colorimetric method; fasting glucose (FG), triglycerides (Tg), total cholesterol (TC) and highdensity lipoprotein (HDL) by enzymatic assays. All analyses were run on a Cobas C501 chemistry analyzer (Roche Diagnostic, Mannheim, Germany). Low-density lipoproteins (LDL) were estimated by Friedewald formula, if $\mathrm{Tg}<400 \mathrm{mg} / \mathrm{dL}(4.52 \mathrm{mmol} / \mathrm{L})(\mathrm{LDL}=\mathrm{TC}-$ $[\mathrm{HDL}+(1 / 5 \mathrm{Tg})])$. Glomerular filtration rate (GFR) was calculated using Chronic Kidney Disease Epidemiology Collaboration (CKD-EPI) equation [22]. A1c was measured by high performance liquid chromatography (HPLC) method (VARIANT II ${ }^{\mathrm{TM}}$ System, Bio-Rad, California, EUA) in K2EDTA samples.

\section{Statistical analysis}

GA levels are expressed as \% and categorized by gender and age. Reference intervals of GA were expressed as $2.5^{\text {th }}$ to $97.5^{\text {th }}$ percentile. Other values were expressed as mean \pm standard deviation (SD), median (interquartile interval) or frequencies (\%) as appropriate. Shapiro-Wilk test was used to verify the normality of variables. Pearson's correlation was applied to analyze the association between variables with both normal distribution and linear correlation, according to the scatter plot. When the criteria to use Pearson's coefficient was not fulfilled, Spearman's coefficient was calculated. Independent-Samples $\mathrm{T}$ test was used to compare GA levels between genders and body mass index (BMI) categories $\left(<\right.$ and $\geq 25 \mathrm{~kg} / \mathrm{m}^{2}$ ), and One-way ANOVA to evaluate GA between age groups defined by quartile intervals. Statistical analysis was performed using the Statistical Package for Social Sciences ${ }^{\circledR}$ (SPSS, version 20.0) software and results were considered as statistically significant when $\mathrm{P}<0.05$.

\section{Results}

One hundred thirty-six apparently healthy individuals were enrolled. Their laboratory and demographic characteristics are presented in table I.

GA levels ranged from $11.1 \%$ to $17.5 \%\left(2.5^{\text {th }}\right.$ and $97.5^{\text {th }}$ percentiles, respectively). The values of GA stratified according to gender and age are presented in table II. 
Table I. General features of the study participants.

\begin{tabular}{|c|c|}
\hline $\mathbf{N}$ & 136 \\
\hline Age (years) & $33(18-74)$ \\
\hline Female, N (\%) & $81(60)$ \\
\hline Caucasians, N (\%) & $125(92)$ \\
\hline $\mathrm{FG}(\mathrm{mmol} / \mathrm{L})$ & $4.9 \pm 0.5$ \\
\hline$(\mathrm{mg} / \mathrm{dL})$ & $88.0 \pm 9.0$ \\
\hline A1c $(\%)$ & $5.3 \pm 0.2$ \\
\hline$(\mathrm{mmol} / \mathrm{mol})$ & $34 \pm 2.2$ \\
\hline Albumin $(\mathrm{g} / \mathrm{L})$ & $43.9 \pm 3.7$ \\
\hline$(\mathrm{g} / \mathrm{dL})$ & $4.4 \pm 0.4$ \\
\hline BMI $\left(\mathrm{kg} / \mathrm{m}^{2}\right)$ & $23.7(21.4-25.7)$ \\
\hline $\mathrm{Tg}(\mathrm{mmol} / \mathrm{L})$ & $1.0(0.8-1.5)$ \\
\hline$(\mathrm{mg} / \mathrm{dL})$ & $88.5(70.8-132.7)$ \\
\hline $\mathrm{TC}(\mathrm{mmol} / \mathrm{L})$ & $4.7 \pm 1.0$ \\
\hline$(\mathrm{mg} / \mathrm{dL})$ & $181.5 \pm 38.6$ \\
\hline $\operatorname{HDL}(\mathrm{mmol} / \mathrm{L})$ & $1.4 \pm 0.4$ \\
\hline$(\mathrm{mg} / \mathrm{dL})$ & $54.1 \pm 15.4$ \\
\hline LDL (mmol/L) & $2.7 \pm 0.9$ \\
\hline$(\mathrm{mg} / \mathrm{dL})$ & $104.2 \pm 34.7$ \\
\hline Creatinine $(\mu \mathrm{mol} / \mathrm{L})$ & $73.4 \pm 15.9$ \\
\hline$(\mathrm{mg} / \mathrm{dL})$ & $0.83 \pm 0.18$ \\
\hline $\operatorname{GFR}\left(\mathrm{mL} / \mathrm{min} / 1.73 \mathrm{~m}^{2}\right)$ & $101.3 \pm 18.9$ \\
\hline
\end{tabular}

FG: fasting glucose; A1c: glycated hemoglobin; BMI: body mass index; Tg: triglycerides; TC: total cholesterol; HDL: high density lipoprotein; LDL: low density lipoprotein; GFR: glomerular filtration rate. Data are presented as mean $\pm \mathrm{SD}$, median (interquartile interval) or median (min-max; for age). not differ between groups ( $\mathrm{p}=0.396)$. Between the BMI groups, there was no difference in age or gender. As most of the volunteers were Caucasians, we did not perform a comparison between GA and ethnic groups.

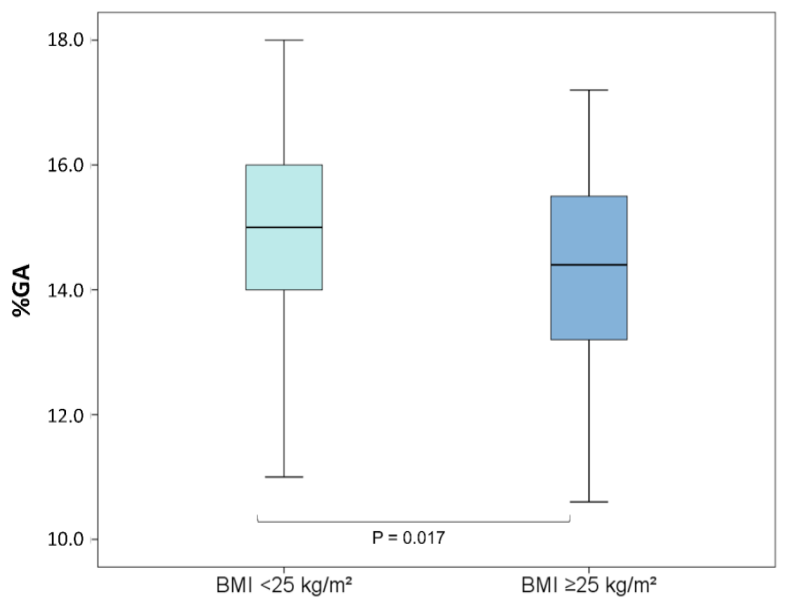

Figure 1. Box-plot of GA levels stratified by BMI $<25 \mathrm{~kg} /$ $\mathrm{m}^{2}(\mathrm{~N}=91)$ and $\geq 25 \mathrm{~kg} / \mathrm{m}^{2}(\mathrm{~N}=45)$. P-value was obtained by Independent-Samples T test.

GA showed a weak to moderate statistically significant correlation with A1c $(\mathrm{R}=0.34, \mathrm{p}<0.001)$, but no correlation was found to $\mathrm{GA}$ and $\mathrm{FG}, \mathrm{A} 1 \mathrm{c}$ and $\mathrm{FG}$, nor to $\mathrm{GA} / \mathrm{A} 1 \mathrm{c}$ and $\mathrm{FG}$. GA and $\mathrm{GA} / \mathrm{A} 1 \mathrm{c}$ ratio were weakly negatively correlated with BMI (Figure 2) $(\mathrm{R}=-0.25, \mathrm{p}=0.004 ; \mathrm{R}=-0.27, \mathrm{p}=0.001$, respectively $)$. Among the glycemic markers, only GA/A1c ratio presented correlation with Tg levels $(\mathrm{R}=-0.27, \mathrm{p}=0.002)$. No correlation was found between the glycemic markers and TC, HDL, LDL, creatinine, or GFR.

Table II. GA levels in normoglycemic Southern Brazilians categorized by gender and age.

\begin{tabular}{l|c|c|c|c|c|c|c|c|c|}
\hline & All & Males & Females & P & Age 1 & Age 2 & Age 3 & Age 4 & p \\
N & 136 & $55(40 \%)$ & $81(60 \%)$ & & $35(26 \%)$ & $35(26 \%)$ & $35(26 \%)$ & $31(22 \%)$ \\
GA Mean (\%) & 14.7 & 14.4 & 14.9 & 0.087 & 15.0 & 14.7 & 14.4 & 14.7 & 0.516 \\
SD & 1.6 & 1.7 & 1.5 & & 1.4 & 1.8 & 1.5 & 1.6 \\
IC 95\% & $14.4-15.0$ & $14.0-14.9$ & $14.6-15.2$ & $14.5-15.5$ & $14.1-15.3$ & $13.9-15.0$ & $14.1-15.3$ \\
$\mathbf{5}^{\text {th }} \mathbf{p}$ & 11.5 & 11.2 & 12.1 & 12.8 & 11.2 & 11.0 & 11.9 \\
$\mathbf{1 0}^{\text {th }} \mathbf{p}$ & 12.6 & 12.0 & 12.9 & 13.0 & 11.4 & 12.6 & 12.6 \\
$\mathbf{9 0}^{\text {th }} \mathbf{p}$ & 16.9 & 16.8 & 17.1 & 17.2 & 16.8 & 16.6 & 17.0 \\
$\mathbf{9 5}^{\text {th }} \mathbf{p}$ & 17.2 & 17.2 & 17.3 & 17.4 & 17.4 & 17.1 & 17.6 \\
Amplitude & 7.4 & 7.4 & 7.0 & 5.3 & 7.0 & 6.7 & 6.5
\end{tabular}

Age 1: 18 to 26 years; Age 2: 27 to 33 years; Age 3: 34 to 48 years; Age 4: 49 to 74 years; SD: standard deviation; IC: $95 \%$ confidence interval for mean; p: percentile; Amplitude: maximum GA value - minimum GA value. Values are expressed as \%GA. P-value was obtained by independent samples T-test or One-Way ANOVA. 

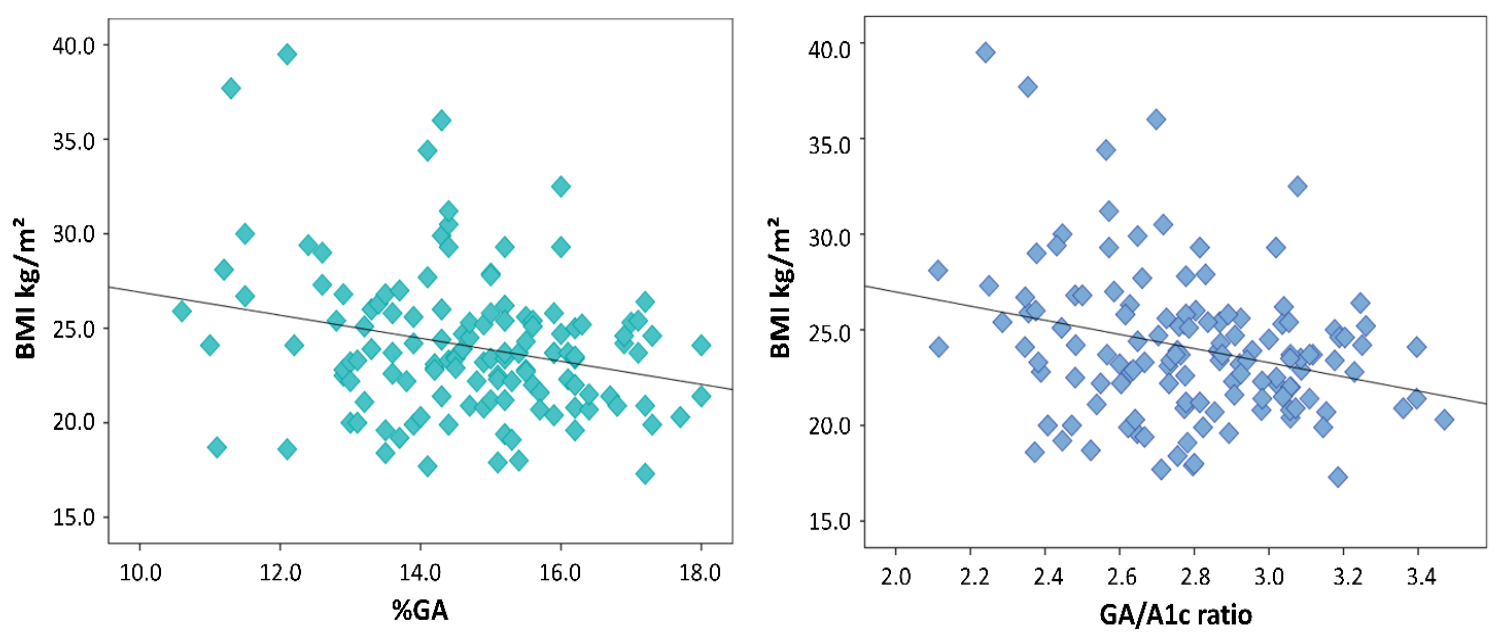

Figure 2. Correlations between $\mathrm{GA}(\mathrm{R}=-0.25, \mathrm{p}=0.004)$ and $\mathrm{GA} / \mathrm{A} 1 \mathrm{c}$ ratio $(\mathrm{R}=-0.27, \mathrm{p}=0.001)$ versus $\mathrm{BMI}$.

\section{Discussion}

This study evaluated GA levels and the associated factors in Southern Brazilians without diabetes or prediabetes. GA levels presented herein are similar to those reported in other populations worldwide. GA values were lower in overweight/obese individuals, and not affected by age or gender. GA/A1c ratio showed a statistically significant weak correlation to BMI and $\mathrm{Tg}$.

At present, enzymatic assays to measure GA are well standardized and have been automated for high

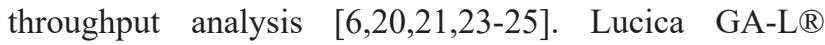
was the first enzymatic methodology launched on the market [8]. It is currently the method most employed for diabetes management and in clinical research in Asian countries [7,10,24]. Nevertheless, GlycoGap ${ }^{\circledR}$ kit is the only enzymatic assay that has been approval by the Food and Drug Administration in the United States (USA) [10] and The Brazilian National Health Surveillance Agency. However, GA results measured by GlycoGap ${ }^{\circledR}$ and Lucica GA-L ${ }^{\circledR}$ have shown a good correlation and concordance $[20,23]$.

There are only two studies that assessed the levels of GA in adults without diabetes using GlycoGap ${ }^{\circledR}$ GA kit. Abidin et al. found an interval of $10.4 \%$ to $15.7 \%$ ( $=$ 130) [20], whereas Rodriguez-Capote et al. reported GA levels ranging from $10.5 \%$ to $17.9 \%(n=44)$ [23]. The first one studied African Americans [20], and the second is a Canadian study [23] that did not provide information about the ethnic group of the participants. The slight differences observed between our results in Southern Brazilians with those in the literature, using the same methodology, might be explained by demographic and ethnic disparities [16]. Moreover, our results are similar to those obtained with
Lucica GA-L $\AA$ method, regardless of the population and/ or ethnicity studied $[5,6,12-14,17,25,26]$.

In subjects without diabetes, it seems there is a moderate fluctuation in the GA/A1c ratio, ranging from 1.9 to 2.7 in Japanese, Chinese and Korean individuals $[5,11,19,27]$. In our study, we found a higher GA/A1c ratio, around $2.8 \pm 0.3$. However, Asian individuals present higher A1c values when compared to Caucasians [15]. Increased A1c levels may account for decreased GA/A1c ratio and should be considered when different ethnicities are evaluated.

It is well known that there are ethnic differences in A1c levels and that African-Americans have higher A1c than Caucasians $[15,16]$. Some investigations have already demonstrated the same pattern in GA results between AfricanAmericans and Caucasians [6,16], but no comparisons have been shown for other ethnic groups. In our study we did not evaluate GA levels considering the ethnicity, due to the very high prevalence of Caucasians in our study. This corroborates with the ethnic distribution in Southern Brazil regarding government agencies' reports [28].

We did not observe differences in GA results regarding gender. This is a conflicting issue in the literature. Kohzuma et al. [6] and Furusyo et al. [5] reported similar GA results in males and females, although Araki et al. [24] and Ikezaki et al. [18] found higher GA levels in Japanese women, same as Zhou et al. [14] in Chinese females. On the other hand, Hsu et al. [13] described higher GA levels only in Taiwan women without diabetes, when compared to those with diabetes. Our results showed similar GA values for men and women.

It is known that GA is lower in infants than in adults due to both plasma glucose and protein levels that are lower 
in infants. However, pathological conditions that influence albumin metabolism might affect GA levels independently of age group [10]. In this study, no differences were observed in GA levels regarding age, which was expected, since we evaluated only apparently healthy adult volunteers. However, Furusyo et al. [5] and Araki et al. [24] reported that GA was higher in older individuals. Besides, Wu et al. [9] have also found that GA is positively associated with age. These discordant results could be explained by the selection criteria of these studies, which did not exclude patients with prediabetes, while our study evaluated only normoglycemic individuals. Therefore, it may be speculated that these higher GA levels in older subjects were due to an increase in plasma glucose itself.

In this study, overweight/obese volunteers (BMI $\geq 25 \mathrm{~kg} / \mathrm{m}^{2}$ ) presented lower GA levels, corroborating with the negative correlation found between GA and GA/A1c ratio with BMI. Similar findings were previously reported $[18,29,30]$. Hsu et al. evaluated the effect of BMI on GA/ A1c ratio according to glucose tolerance status [13] and reported that individuals with normal glucose tolerance presented the strongest inverse correlation between BMI and GA/A1c ratio, and it was not influenced by any other variable. However, in prediabetes and type 2 diabetes groups, this correlation was respectively weaker, and GA/ A1c ratio showed to be primarily influenced by FG and postprandial serum glucose levels rather than BMI. These results suggest that, even though body weight influences negatively GA levels, overweight/obese subjects in a prediabetes state may be accurately monitored by GA/ A1c ratio [13]. Additionally, He et al. recently described an independent and negative association between GA and GA/A1c levels with BMI, in both patients with and without diabetes. They showed that for every $1 \mathrm{~kg} / \mathrm{m}^{2}$ increment in BMI, GA absolute values decreased by nearly $0.13 \%$ [30]. Furthermore, it has been hypothesized that an increase in fat mass, in addition to the high release of inflammatory adipokines, leads to lower albumin synthesis and raised albumin turnover $[18,29]$. Besides, the effect of insulin on albumin turnover is unclear [29]. Indeed, the mechanisms that explain the relationship between GA and obesity is a question still open to debate.

Normoglycemic participants in this study showed a weak correlation between GA and A1c, but no association was seen with FG. Such data in subjects without diabetes are rare in literature to allow any comparison. However, since healthy individuals are supposed to have low glucose variations, a weak association between GA and A1c may be expected. Tg levels were negatively correlated to GA/ A1c, which is in agreement with Park's et al. findings [11].

One limitation of this study was the relatively small sample size, although in agreement with international recommendations [31]. Another drawback was that we evaluated the presence of hypothyroidism, hyperthyroidism, and cirrhosis only by self-report. On the other hand, the participants were carefully selected to exclude those with prediabetes or diabetes. Besides, as far as we know, this study is the first to provide information about GA levels in Brazilians and to explore its associated factors of variation.

\section{Conclusions}

In conclusion, GA levels in Brazilians were similar to those previously described in other populations and/or ethnicities. However, ethnicity, lifestyle, and nutritional habits differences may account for the slight discrepancies in GA values among different regions worldwide. Our data show that GA values are irrespective of sex or age and present a weak correlation with weight in normoglycemic subjects. These factors should be considered in the interpretation of GA results.

\section{Aknowledgements}

This work was supported by the Research Incentive Fund (FIPE) of the Hospital de Clínicas de Porto Alegre (HCPA) (FIPE/HCPA, GPPG 130440). PACF received scholarship from Programa de Excelência Acadêmica da Coordenação de Aperfeiçoamento de Pessoal de Nível Superior - Brasil (CAPES-PROEX). MKH received a undergraduate scholarship from Conselho Nacional de Desenvolvimento Científico e Tecnológico (CNPq).

\section{References}

1. International Diabetes Federation. IDF Diabetes Atlas, 8th edn. Brussels, Belgium: International Diabetes Federation 2017. Available from: http://www.diabetesatlas.org.

2. American Diabetes Association. Classification and Diagnosis of Diabetes: Standards of Medical Care in Diabetes-2020. Diabetes Care. 2020;43(Suppl 1):S14-S31.

3. Nathan DM, McGee P, Steffes MW, Lachin JM; DCCT/EDIC Research Group. Diabetes. Relationship of glycated albumin to blood glucose and $\mathrm{HbAlc}$ values and to retinopathy, nephropathy, and cardiovascular outcomes in the DCCT/ EDIC study. Diabetes. 2014;63:282-290.

4. Cavagnolli G, Pimentel AL, Freitas PA, Gross JL, Camargo JL. Factors affecting A1C in non-diabetic individuals: Review and meta-analysis. Clin Chim Acta. 2015;445:107-114.

5. Furusyo N, Koga T, Ai M, Otokozawa S, Kohzuma T, Ikezaki H, et al. Utility of glycated albumin for the diagnosis of diabetes mellitus in a Japanese population study: results from the Kyushu and Okinawa Population Study (KOPS). Diabetologia. 2011;54:3028-3036.

6. Kohzuma T, Yamamoto T, Uematsu Y, Shihabi ZK, Freedman BI. Basic performance of an enzymatic method for glycated albumin and reference range determination. J Diabetes Sci Technol. 2011;5:1455-1462.

7. Freitas PAC, Ehlert LR, Camargo JL. Glycated albumin: a potential biomarker in diabetes. Arch Endocrinol Metab. 2017;61:296-304.

8. Kouzuma T, Usami T, Yamakoshi M, Takahashi M, Imamura 
S. An enzymatic method for the measurement of glycated albumin in biological samples. Clin Chim Acta. 2002;324:6171.

9. Wu WC, Ma WY, Wei JN, Yu TY, Lin MS, Shih SR, et al. Serum Glycated Albumin to Guide the Diagnosis of Diabetes Mellitus. PLoS One. 2016;11:e0146780.

10. Dozio E, Di Gaetano N, Findeisen P, Corsi Romanelli MM. Glycated albumin: from biochemistry and laboratory medicine to clinical practice. Endocrine. 2017;55:682-690.

11. Park S, Lee W, Chung HS, Hong KS. Diagnostic Utility of Serum Glycated Albumin for Diabetes Mellitus and Its Correlation With Hyperlipidemia. Ann Lab Med. 2016;36:306-312.

12. Tominaga M, Makino H, Yoshino G, Kuwa K, Takei I, Aono $\mathrm{Y}$, et al. Report of the committee on standardization of laboratory testing related to diabetes mellitus of Japan Diabetes Society: determination of reference intervals of hemoglobin A1c (IFCC) and glycoalbumin in the Japanese population. Journal of the Japan Diabetes Society. 2006;49:825-833.

13. Hsu P, Ai M, Kanda E, Yu NC, Chen HL, Chen HW, et al. A comparison of glycated albumin and glycosylated hemoglobin for the screening of diabetes mellitus in Taiwan. Atherosclerosis. 2015;242:327-333.

14. Zhou Q, Shi DB, Lv LY. The establishment of biological reference intervals of nontraditional glycemic markers in a Chinese population. J Clin Lab Anal. 2017;31:e22097.

15. Cavagnolli G, Pimentel AL, Freitas PAC, Gross JL, Camargo JL. Effect of ethnicity on HbA1c levels in individuals without diabetes: Systematic review and meta-analysis. PLoS One. 2017; 12:e0171315.

16. Parrinello CM, Sharrett AR, Maruthur NM, Bergenstal RM, Grams ME, Coresh J, et al. Racial Differences in and Prognostic Value of Biomarkers of Hyperglycemia. Diabetes Care. 2016;39:589-595.

17. Testa R, Ceriotti F, Guerra E, Bonfigli AR, Boemi M, Cucchi $\mathrm{M}$, et al. Glycated albumin: correlation to HbAlc and preliminary reference interval evaluation. Clin Chem Lab Med. 2017;55:e31-e33.

18. Ikezaki H, Furusyo N, Ihara T, Hayashi T, Ura K, Hiramine S, et al. Glycated albumin as a diagnostic tool for diabetes in a general Japanese population. Metabolism. 2015;64:698-705.

19. Huh JH, Kim KJ, Lee BW, Kim DW, Kang ES, Cha BS, et al. The relationship between BMI and glycated albumin to glycated hemoglobin (GA/A1c) ratio according to glucose tolerance status. PLoS One. 2014;9:e89478.
20. Abidin D, Liu L, Dou C, Datta A, Yuan C. An improved enzymatic assay for glycated serum protein. Anal Methods. 2013;5:2461-2469.

21. Freitas PAC, Ehlert LR, Camargo JL. Comparison between two enzymatic methods for glycated albumin. Analytical Methods. 2016;8:8173-8178.

22. Levey AS, Stevens LA, Schmid CH, Zhang Y, Castro AF 3rd, Feldman HI, et al. A new equation to estimate glomerular filtration rate. Ann Intern Med. 2009;150:604-612.

23. Rodriguez-Capote K, Tovell K, Holmes D, Dayton J, Higgins TN. Analytical evaluation of the diazyme glycated serum protein assay on the siemens ADVIA 1800: comparison of results against hbalc for diagnosis and management of diabetes. J Diabetes Sci Technol. 2015;9:192-199.

24. Araki T, Ishikawa Y, Okazaki H, Tani Y, Toyooka S, Satake $\mathrm{M}$, et al. Introduction of glycated albumin measurement for all blood donors and the prevalence of a high glycated albumin level in Japan. J Diabetes Investig. 2012;3:492-497.

25. Paleari R, Bonetti G, Callà C, Carta M, Ceriotti F, Di Gaetano $\mathrm{N}$, et al. Multicenter evaluation of an enzymatic method for glycated albumin. Clin Chim Acta. 2017;469:81-86.

26. Sumner AE, Duong MT, Aldana PC, Ricks M, TullochReid MK, Lozier JN, et al. A1C Combined With Glycated Albumin Improves Detection of Prediabetes in Africans: The Africans in America Study. Diabetes Care. 2016;39:271-277.

27. Mo Y, Ma X, Li H, Ran X, Yang W, Li Q, et al. Relationship between glycated albumin and glycated hemoglobin according to glucose tolerance status: A multicenter study. Diabetes Res Clin Pract. 2016;115:17-23.

28. Instituto Brasileiro de Geografia e Estatística (IBGE), Censo demográfico 2010 - Características gerais da população, religião e pessoas com deficiência. Rio de Janeiro, RJ: IBGE. 2010:1-215.

29. Reynolds AN, Duncan A, Kruimer D, Venn BJ. Glycated albumin is associated with body mass index in euglycemic adults but is not predictive of postprandial blood glucose response. J Clin Lab Anal. 2017;31:e22085.

30. He X, Mo Y, Ma X, Ying L, Zhu W, Wang Y, et al. Associations of body mass index with glycated albumin and glycated albumin/glycated hemoglobin $\mathrm{A}_{1 \mathrm{c}}$ ratio in Chinese diabetic and non-diabetic populations. Clin Chim Acta. 2018;484:117-121.

31. Clinical and Laboratory Standards Institute (CLSI). EP28A3C: Defining, Establishing, and Verifying Reference Intervals in the Clinical Laboratory; Approved Guideline Third Edition. Wayne, PA: CLSI. 2010:1-72. 